\title{
MODELS OF STRESS PROPAGATION IN GRANULAR MEDIA
}

\author{
J.P. BOUCHAUD AND P. CLAUDIN \\ Service de Physique de l'Etat Condensé, \\ CEA, Ormes des Merisiers, \\ 91191 Gif-sur-Yvette, Cedex France.
}

AND

M. E. CATES AND J. P. WITTMER

Dept. of Physics and Astronomy, University of Edinburgh JCMB King's Buildings, Mayfield Road, Edinburgh EH9 3JZ, UK.

\begin{abstract}
Stress patterns in static granular media exhibit unusual features when compared to either liquids or elastic solids. Qualitatively, we attribute these features to the presence of 'stress paths', whose geometry depends on the construction history and controls the propagation of stresses. Stress paths can cause random focussing of stresses (large fluctuations) as well as systematic deflections (arching). We describe simple physical models that capture some of these effects. In these models, the 'stress paths' become identified with the characteristic 'light rays' of wavelike (hyperbolic) equations for force propagation. Such models account for the 'pressure dip' below conical sandpiles built by pouring from a point source, and explain qualitatively the large stress fluctuations observed experimentally in granular matter. The differences between this approach and more conventional modelling strategies (based on elastoplastic or rigid-plastic models) are highlighted, focusing on the role of boundary conditions. Our models provide a continuum picture in which granular materials are viewed as fragile matter, able to support without rearranging only a subset of the static external loadings admissible for a normal elastic solid.
\end{abstract}




\section{Introduction}

Stress patterns in granular media exhibit some rather unusual features when compared to either liquids or elastic solids. For example, the vertical pressure below conical sandpiles does not follow the height of material above a particular point, but rather has a minimum underneath the apex of the pile $[1,2,3]$. Furthermore, local stress fluctuations are large, sometimes on length scales much larger than the grain size. For example, repeatedly pouring the very same amount of powder in a silo results in fluctuations of the weight supported by the bottom plate of $20 \%$ or more [4, 5]. Qualitatively, these features are attributed to the presence of stress paths which can focus the stress field into localized regions and also deflect it to cause "arching" (see also [6, 7], and [8] for early qualitative experiments).

More quantitative experiments were recently performed by Liu et al. [9], Brockbank et al. [2] and Mueth et al. [10], where the local fluctuations of the normal stress deep inside a silo or at the base of a sandpile were measured. It was found that the stress probability distribution is rather broad, decaying exponentially for large stresses. A simple 'scalar' (one component) model for stress propagation was introduced and studied in detail [9, 11], which predicts a stress probability distribution in good agreement with experimental (and numerical) data. However, this model only considers the vertical normal component of the stress tensor, and discards all the other components.

A fully 'tensorial' model for stress propagation in homogeneous granular media was proposed in $[12,3,13]$ to account for the pressure 'dip' described above. The most striking feature of this model is that the stress propagation equation is (at least in two dimensions) a wave equation, with the vertical axis playing the rôle of time. In this model, 'stress paths' naturally appear as the characteristics - or the 'light rays' - of the corresponding hyperbolic equation. Note that the standard equations of elasticity are elliptic; the fundamental difference between these two cases will be discussed later. Both must also be contrasted with the scalar model, which corresponds to a parabolic equation, and in which stresses travel almost vertically.

The aim of this paper is to review some of the recent theoretical work in this field. We will start by summarizing the content of the scalar ' $q$-model', which, although unsatisfactory in several respects, offers the advantage of simplicity. Keeping the spirit of the ' $q$-model', we then show that the introduction of the shear stress fundamentally modifies the structure of the equations and leads to wave-like propagation. Several issues concerning the solution of this wave equation are then discussed. We then consider some variants of the wave equation, in particular to account for local inhomogeneities or for anisotropy, for example induced by the construction history. 
(The importance of construction history in granular matter has been acknowledged for at least a century [14].) Among a family of models, the 'Fixed Principal Axis' (FPA) limit plays a special rôle which we discuss in relation with experimental data on conical sandpiles. We then consider in more detail the differences between this approach and some more conventional modelling strategies (based on elastoplastic or rigid-plastic models), and try to shed some light on the controversy that the "hyperbolic" approach to sandpile modelling has raised recently $[15,16,17]$. This discussion focuses in particular on the rôle of boundary conditions.

Much of our discussion will, for clarity, be limited to two dimensional piles. However, most of the recent experimental results are in three dimensions; indeed it was in this context that the physical assumptions of Refs. $[3,13]$ were made.

\section{The Scalar Model}

The main assumption of the scalar model is that only the vertical normal component of the stress tensor $w=\sigma_{z z}$ (the 'weight') needs to be considered. Supposing for simplicity that the grains reside on the nodes of a two-dimensional lattice (see figure 1), the simplest model for weight propagation is:

$$
w(i, j)=w_{g}+q_{+}(i-1, j-1) w(i-1, j-1)+q_{-}(i+1, j-1) w(i+1, j-1)
$$

where ' $w_{g}$ ' is the weight of a grain, and $q_{ \pm}(i, j)$ are 'transmission' coefficients giving the fraction of weight which the grain $(i, j)$ transmits to its right (resp. left) neighbour immediately below. Mass conservation imposes that $q_{+}(i, j)+q_{-}(i, j)=1$ for all $i, j$ 's. The case of an ordered pile of identical grains would correspond to $q_{ \pm}=\frac{1}{2}$. In this case, the equation (1) describes the 'time'-evolution (along the $j$ direction) of the probability density for a random walker on a line. The authors of $[9,11]$ then propose to take into account the local disorder in packing, grain sizes and shapes, etc., by choosing $q_{+}(i, j)$ to be independent random numbers (subject to the above constraint), for example uniformly distributed between 0 and 1 . This case is interesting because it leads to an exact solution for the local weight distribution $P(w)$, in the limit $j \rightarrow \infty$ :

$$
P(w)=\frac{w}{W^{2}} \exp -\frac{w}{W}
$$

where $2 W=j w_{g}$ is the average weight. Liu et al. $[9,11]$ have argued that the exponential tail for large $w$ is generic; however, if the maximum value of $q$ is $q_{M}<1$, one can show that $P(w)$ decays faster than an exponential:

$$
\log P(w) \propto_{w \rightarrow \infty}-w^{\beta} \quad \beta=\frac{\log 2}{\log 2 q_{M}}
$$




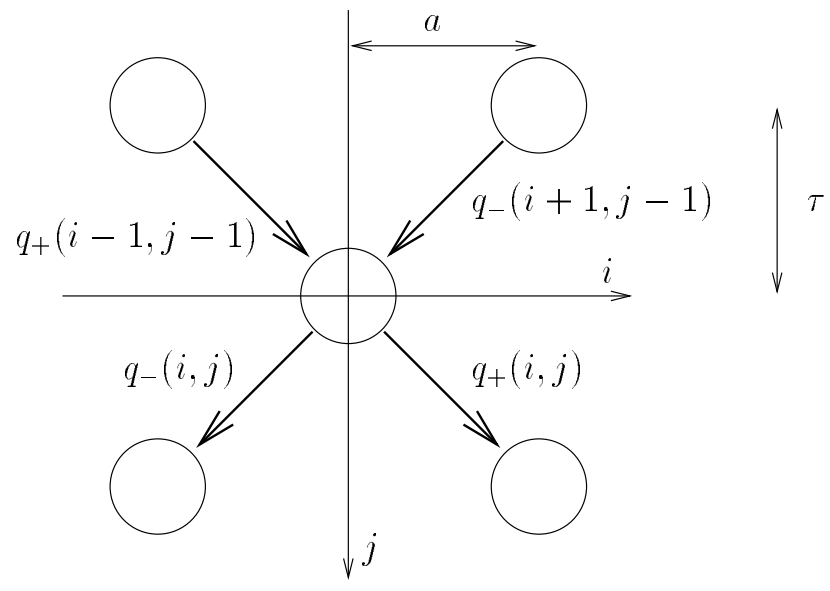

Figure 1. The 'q-model' model with two neighbours. $q_{ \pm}$'s are independent random variables, satisfying the weight conservation constraint: $q_{+}(i, j)+q_{-}(i, j)=1$.

(Notice that $\beta=1$ whenever $q_{M}=1$, and that $\beta=\infty$ in the ordered case $\left.q_{M}=1 / 2\right)$. In this sense the exponential tail of $P^{*}(w)$ is not universal: it requires the possibility that one of the $q$ can be arbitrarily close to 1 , i.e. that there is a non zero probability that one grain is entirely bearing on one of its downward neighbours (local arching).

In the continuum limit, the $q$-model is equivalent to a diffusion equation with a random convection term (related to $q_{+}-q_{-}$), for which several results are known. However, on large scales, the random convection term merely renormalises the diffusion constant; hence, in this model, the response to a localised overload at the top of a pile spreads out diffusively as $\sqrt{D H}$, where $H$ is the height of the pile and $D$ is the diffusion constant, which is of the order of the grain size $a$. In the limit $H \gg a$, the spreading is negligible, which means that the weight essentially propagates vertically. Hence, the $q$-model predicts a 'hump' in the pressure profile underneath a sandpile, directly reflecting its shape.

A way to accomodate a "pressure dip" within this scalar picture was suggested by Edwards (although in a slightly different language). Consider for example a sandpile built from a point source: the history of the grains will certainly inprint a certain oriented 'texture' to the contact network, which can be modelled, within the present scalar model, as a nonzero mean value of the 'convection term', the sign of which depends on which side of the pile is chosen. Let us call $V_{0}$ the average value of this term on the $x \geq 0$ side of the pile, with $-V_{0}$ on the other side. The differential equation 
describing propagation now reads, in the absence of disorder:

$$
\partial_{t} w+\partial_{x}\left[V_{0} \operatorname{sign}(x) w\right]=\rho+D_{0} \partial_{x x} w
$$

Solving this equation in a sandpile geometry leads to a weight minimum around $x=0$. Equation (4) gives a precise mathematical content to Edwards' idea of arching in sandpiles [18], as the physical mechanism leading to a 'dip' in the pressure distribution [1]. As discussed below (see also $[3,13])$, this can be taken much further within a tensorial framework. Let us also note that (4) can in fact be obtained naturally within an extended $q$-model model, with an extra rule accounting for the fact that a grain can slide and lose contact with one of its two downward neighbours [19]. However, this extra sliding rule implicitly refers to the existence of shear stresses, absent in the scalar model. It is more satisfactory to recognize from the outset that stress has a tensorial, rather than scalar, nature. Models which do this are decribed next.

\section{A Tensorial Model}

\subsection{THE WAVE EQUATION}

It is useful to start with a simple toy model for stress propagation, which is the analogue of the model presented in figure 1. We now consider the case of three downward neighbours (see figure 2), for a reason which will become clear below. Each grain transmits to its downward neighbours not

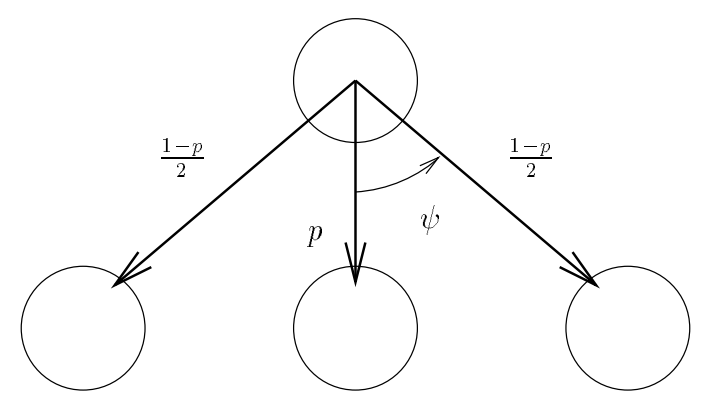

Figure 2. Three neighbour configuration. Each grain tranmits two force components to its downward neighbours. A fraction $p$ of the vertical component is transmitted through the middle leg.

one, but two force components: one along the vertical axis $t$ and one along $x$, which we call respectively $F_{t}(i, j)$ and $F_{x}(i, j)$. For simplicity, we assume that each 'leg' emerging from a given grain can only transport the force parallel to itself (but more general rules could be invented). Assuming that 
the transmission rules are locally symmetric, and that a fraction $p \leq 1$ of the vertical component travels through the middle leg, we find:

$$
\begin{aligned}
F_{x}(i, j)= & \frac{1}{2}\left[F_{x}(i-1, j-1)+F_{x}(i+1, j-1)\right] \\
& +\frac{1}{2}(1-p) \tan \psi\left[F_{t}(i-1, j-1)-F_{t}(i+1, j-1)\right] \\
F_{t}(i, j)= & w_{0}+p F_{t}(i, j-1)+\frac{1}{2}(1-p)\left[F_{t}(i-1, j-1)+F_{t}(i+1, j-1)\right] \\
& +\frac{1}{2 \tan \psi}\left[F_{x}(i-1, j-1)-F_{x}(i+1, j-1)\right]
\end{aligned}
$$

where $\psi$ is the angle between grains, defined in figure 2. Taking the continuum limit of the above equations leads to:

$$
\begin{aligned}
\partial_{t} F_{t}+\partial_{x} F_{x} & =\rho \\
\partial_{t} F_{x}+\partial_{x}\left[c_{0}^{2} F_{t}\right] & =0
\end{aligned}
$$

where $c_{0}^{2} \equiv(1-p) \tan ^{2} \psi$. Eliminating (say) $F_{x}$ between the above two equations leads to a wave equation for $F_{t}$, where the vertical coordinate $t$ plays the rôle of time and $c_{0}$ is the equivalent of the 'speed of light'. In particular, the stress does not propagate vertically, as it does in the scalar model, but rather along two rays, each at a non zero angle $\pm \varphi$ such that $c_{0}=\tan \varphi$. Note that $\varphi \neq \psi$ in general (unless $p=0$ ); the angle at which stress propagates has nothing to do with the underlying lattice structure and can take any value depending on the local rules for force transmission. We chose a three-leg model to illustrate this particular point.

The above derivation can be reformulated in terms of classical continuum mechanics as follows. Considering all stress tensor components $\sigma_{i j}$, the equilibrium equation reads,

$$
\begin{aligned}
\partial_{t} \sigma_{t t}+\partial_{x} \sigma_{x t} & =\rho \\
\partial_{t} \sigma_{t x}+\partial_{x} \sigma_{x x} & =0
\end{aligned}
$$

Identifying the local average of $F_{t}$ with $\sigma_{t t}$ and that of $F_{x}$ with $\sigma_{t x}$, we see that the above equations $(7,8)$ are actually identical to $(9,10)$ provided $\sigma_{t x}=\sigma_{x t}$ (which corresponds to the absence of local torque) and

$$
\sigma_{x x}=c_{0}^{2} \sigma_{t t}
$$

This relation between normal stresses was postulated in [12] as the simplest "constitutive relation" among stress components, obeying the correct symmetries, that one can possibly assume. The term "constitutive relation" 
normally refers to a relation between stress and strain, but the model under discussion has no strain variables defined; instead the particles are viewed as completely rigid. (Equations $(9,10)$ are then indeterminate unless a further hypothesis relating the stresses themselves is made.) This particular choice can be interpreted as a local Janssen approximation [20]. We return later to a more detailed discussion of closure equations of this type. In the present case, the parameter $c_{0}^{2}$ must encode relevant details of the local geometry of the packing (friction, shape of grains, etc.) and may thereby depend on the construction history of the grain assembly. Only for simple, 'homogeneous' histories (such as constructing a uniform sandbed using a sieve) will $c_{0}^{2}$ be everywhere constant on the mesoscopic scale. Even then, unless an ordered packing is somehow created, local fluctuations of $c_{0}^{2}$ will always be present.

\subsection{SOME SIMPLE SITUATIONS}

The simplest situation is that of an infinitely wide layer of sand, of depth $H$, with a localized ( $\delta$-function) overload at the top. The weight at the bottom then defines the response function of the wave equation, which, in two dimensions, is the sum of two $\delta$ peaks localised at $x= \pm c_{0} H$.

Next, one can consider the sandpile geometry. For a pile at repose, the position of the free surfaces are $x= \pm c z$, where $c=\cot \phi$ with $\phi$ the repose angle. On these surfaces, all the stresses vanish. This boundary condition is then (for given $c_{0}$ and $c$ ) sufficient to solve for the stress field everywhere in the pile. (See Section 8 below.) One then find that the vertical normal component of the stress is piecewise linear as a function of $x$. In particular, for $-c_{0} H \leq x \leq c_{0} H, \sigma_{t t}$ is constant. Therefore, in two dimensions, this model [12] predicts a flat-topped stress profile rather than a dip.

For a pile created by depositing grains from above (for example by sieving sand onto a disc) it is natural to expect the free surface to be a slip plane. (This is a plane across which the stress components saturate the Mohr-Coulomb condition.) Interestingly, this provides a relation between $c_{0}$ and the friction angle $\phi$, which reads: $c_{0}^{2}=1 /\left(1+2 \tan ^{2} \phi\right)$ (note that since $c=1 / \tan \phi$, one has automatically $c>c_{0}$ ). Under these conditions one finds that the 'plastic' region (where the Mohr-Coulomb condition is saturated) extend inward from the surface to encompass the outer 'wings' of the pile (i.e. $c_{0} z \leq|x| \leq c z$ ); see figure 3 . This follows from the solution of the model and is not an a priori assumption, of the kind commonly made in elastoplastic modelling (e.g., [16]). In three dimensions, a second closure relation is required [12], but in all cases the stress profile has a broad maximum at the center of the pile. Now, however, the Mohr-Coulomb condition is only saturated in the immediate vicinity of the free surface - 
the 'plastic' region has zero volume in three dimensions $[12,13]$.

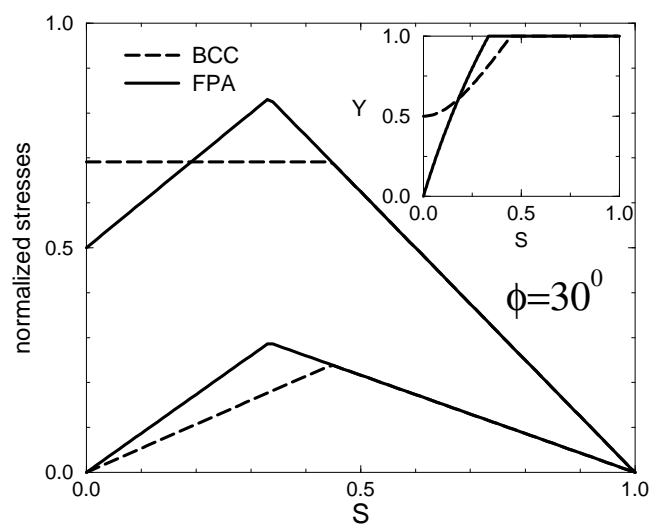

Figure 3. Reduced normal (upper) and shear stress (lower) curves against $S=r \tan \phi / t$ for the symmetric wave equation ("BCC") and the FPA model in two dimensions. Inset: the yield function $Y$. The Mohr-Coulomb inequality is saturated when $Y=1$

\section{Symmetries and Constitutive Relations}

Although above it was motivated in the context of a specific microscopic model, the linear constitutive relation (11) can be viewed, independently of any microscopic model, as the simplest closure equation compatible with the symmetries of the problem. The latter include a local reflection symmetry in which $x-x_{0}$ is changed to $x_{0}-x$ (with $x_{0}$ an arbitrary reflection plane) and also a form of "dilational" symmetry known as RSF ("radial stress field") scaling. RSF scaling depends on the absence of any characteristic stress scale, which follows if the Young's modulus of the grains is sufficiently much larger than any stresses arising in the granular assembly being studied. Such scaling, which requires the stress distributions beneath piles of different heights to have the same shape, is quite well confirmed in some (but not all) experiments on conical sandpiles $[1,2,15]$.

Even with these two symmetries, one can consider more complicated (nonlinear) constitutive relations among stresses, which must be of the form [12]:

$$
\sigma_{x x}=c_{0}^{2} \sigma_{t t} \mathcal{F}\left(\frac{\sigma_{x t}^{2}}{\sigma_{t t}^{2}}\right)
$$

Note that the Mohr-Coulomb condition itself can be written in this form. Viewed as a constitutive equation, it defines a rigid-plastic model whose 
physical content is to assume that, everywhere in the material, a plane can be found across which slip failure is about to occur. (The name "incipient failure everywhere", IFE, aptly describes this model $[12,3,13]$.) All closures of the form (12) lead to a hyperbolic equations for stresses, although in the general case the characteristic directions of propagation (the 'light rays' of the corresponding wave equation) depend on the loading and therefore vary with position.

An interesting situation arises when local reflection symmetry is broken. This is the case, for example, in sandpiles created by pouring from a point source onto a rough surface - which is the usual mode of construction. In such a pile, all grains arriving at the apex of the pile roll (in two dimensions) either to the right or to the left. The two halves of the pile therefore have different construction histories that are mirror images of each other. This violates local reflection symmetry, and in general permits constitutive equations such as:

$$
\sigma_{x x}=c_{0}^{2} \sigma_{t t} \mathcal{G}\left(\frac{\operatorname{sign}(x) \sigma_{x t}}{\sigma_{t t}}\right)
$$

The simplest case (found e.g. by expanding $\mathcal{G}$ to first order in the shear to normal stress ratio) corresponds to a family of (quasi-) linear constitutive relations [13]:

$$
\sigma_{x x}=c_{0}^{2} \sigma_{t t}+\mu \operatorname{sign}(x) \sigma_{x t}
$$

The previous, symmetrical, case has $\mu=0$. For nonzero $\mu$, (14) again leads to a wave equation, although this time anisotropic, in the sense that the two characteristic rays make asymmetric angles to the vertical axis. Note that such a model can be obtained from rules such as those in figure 2 simply by having an asymmetric partitioning of forces between the supporting grains (or indeed by tilting the entire packing). Note also that $x=0$ is a singular line across which the directions of propagation change discontinuously. Microscopically, $\mu \neq 0$ also leads to an unequal sharing of the weight of a grain between the two characteristic rays propagating downward from it. For $\mu<0$, most of the weight travel outwards; this provides, within a fully tensorial model, a mathematical description of the tendency to form arches, as developed by Edwards for the scalar case.

Solving these anisotropic wave equations for sandpiles in two dimensions one again finds for $\sigma_{t t}$ a piecewise linear function, which now has a sharp maximum at $x=0$ when $\mu>0$, but a minimum for $\mu<0$, in accord with the arching scenario mentioned above (see figure 3 ). If one furthermore imposes, as above, that the free surfaces are slip planes, one finds a relation between $c_{0}^{2}, \mu$ and $\phi$.

$$
c_{0}^{2}=\frac{1}{1+2 \tan ^{2} \phi}[1-\mu \tan \phi]
$$


One again finds the result that the material throughout the outer wings of the pile (exterior to the triangle formed by the characteristics passing through the apex) are at incipient (Mohr-Coulomb) failure.

\subsection{THE FPA MODEL}

Among possible forms of (14) there is a particular case, corresponding to $c_{0}^{2}=1$, which has some intriguing special properties:

$$
\sigma_{x x}=\sigma_{t t}-2 \operatorname{sign}(x) \tan (\phi) \sigma_{x t}
$$

(The resulting value of $\mu$ is that appropriate to the boundary conditions stated above.) Specifically in this case, the principal axis of the stress tensor coincide with the 'light rays' and hence have a fixed direction throughout the pile (up to a reflection symmetry across the line $x=0$ ). This particular case was called the FPA ("fixed principal axes") constitutive equation in $[3,13]$. It corresponds to an assumption that the anisotropic texture of the medium imparts a local stress rule which requires that the orientation of the stress tensor is fixed at the time of burial and, thereafter, cannot change under further loading (The values of the stress components themselves can, of course, change.). Since all material elements are buried near the free surface of the pile, where the stress tensor orientation is known the fact that the surface is itself a slip plane [3], this fixes the principal axes throughout the pile. The resulting stresses are shown in figure 3.

At first sight, there is a problem with the FPA description on the symmetry axis of the pile $(x=0)$, where the stress ellipsoid is required simultaneously to have two conflicting orientations. However, since $c_{0}^{2}=1$ the stress is isotropic at the centre, and there is no conflict. However, this shows that the defining feature of the FPA model is very easily lost; as soon as a slightly different $c_{0}^{2}$ is chosen, the principal axes are no longer of fixed orientation but rotate smoothly as one passes from one side of the pile to the other.

\section{Experiments on Cones and Wedges}

We do not have space here for an extended discussion of the experimental data on cones and wedges; this can be found elsewhere [21]. Here we merely summarize some of the most important points.

\subsection{CONES}

The extension of the FPA model to three dimensions, like the other mod-

els discussed above, requires a second constitutive relation among stresses 
to close the problem. Several well-known candidates [22] exist for this secondary closure and give similar results; these results are in surprisingly good agreement with the experimental data for the pressure dip in three dimensional conical sandpiles built by pouring from a point source onto a rough rigid support $[3,13,2]$; see figure 4 .

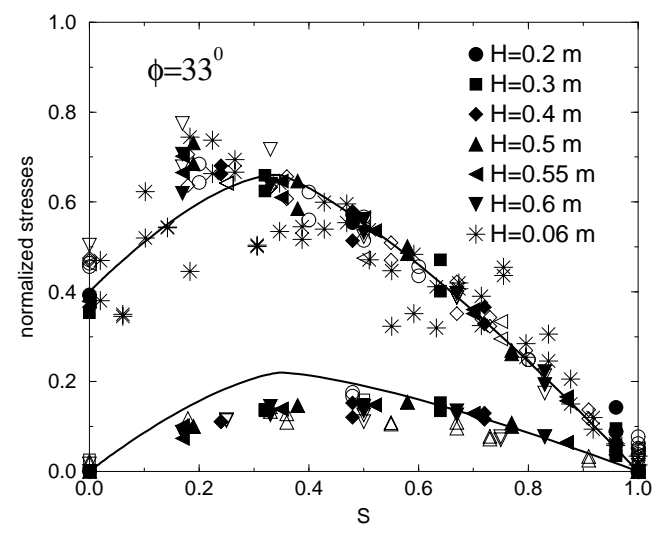

Figure 4. Comparison of FPA model (using a uniaxial secondary closure [12, 3, 13]) with scaled experimental data of Smid and Novosad and $\left(^{*}\right)$ that of Brockbank et al (averaged over three piles). Upper and lower curves denote normal and shear stresses. The data is used to calculate the total weight of the pile which is then used as a scale factor for stresses. The horizontal coordinate is scaled by the pile radius.

We believe that the experimental data strongly suggest an FPA-like model (corresponding to $c_{0}^{2} \simeq 1$, Eq.16), although they do not prove that the principal axes of a material element are necessarily fixed. Of greater physical significance is the idea that the characteristic rays of the wave equation could be fixed at burial. (Below we will connect this with the idea of stress paths.) This is the content of a more general interpretation (the "oriented stress linearity" model of [13]) in which $\mu$ (or $c_{0}^{2}$ ) is a parameter characterising the local anisotropy of the granular packing. This is fixed by construction history, in a manner as yet unspecified; but for a point-source pile values close to the FPA limit are suggested by the data. In contrast, for a pile constructed by sieving, we might expect the local reflection symmetry to be unbroken $(\mu=0)$, leading instead to a smooth maximum in the pressure, as predicted from Eq.11. Experiments on the construction-history dependence of the stress profile would therefore be welcome.

Note that the idea that some property of the medium, represented by a constitutive equation among stresses, is "fixed at burial" is an important 
simplifying assumption of our approach to sandpile modelling. This has been called the "perfect memory" assumption [13].

\subsection{WEDGES: SURFACE AVALANCHES OR DEEP YIELD?}

Savage [15] has pointed out that some classical data on wedges, as opposed to cones, are apparently at odds with these ideas. Such wedges are of triangular cross-section but very long in the third direction, i.e., quasi-two dimensional. According to classical reports [23, 24], the stress distribution beneath such wedges shows little dependence on the construction history, but a very strong dependence on whether the base supporting the wedge is allowed to sag. Without basal sag, almost no pressure dip is reported [15].

At first sight, then, the pressure dip in conical piles might also be attributable to basal sag. We strongly believe that this viewpoint is untenable, especially in the light of the data of Brockbank et al [2] (see figure 4) in which the measurement system leads to discernable localized indentation, rather than a slight curvature (sagging) of the base under regions of high pressure. Moreover, the classical data on wedges is somewhat scant, of dubious accuracy, and in most cases does not specify the construction history of the wedge in any detail. We would therefore encourage renewed experimental investigation of wedges of sand.

There is, in any case, an important difference in the physics of wedges and cones. This concerns the geometry of the "plastic" region (that in which the Mohr-Coulomb inequality is saturated) near the surface of the pile. All our models, including the FPA limit [13], predict that this is infinitely narrow in the three dimensional conical pile, but that for a wedge it extends through a large outer zone (exterior to the two characteristic rays passing through the apex). In the first case, the perfect memory assumption appears selfconsistent: the presence of a thin yield layer at the surface suggests that the pile grows by surface avalanches which do not disturb its internal structure too much. In contrast, the status of the perfect memory assumption is, for a wedge, far less clear. Since a broad zone of marginal instability exists beneath the surface, the surface flow could cause "deep yield" events $[25,17]$ which would disrupt the internal structure of the pile [21]. This could lead to a local isotropisation of the granular texture and cause values of $\mu$ in (14) closer to those of the symmetric propagation model (Eq.11) than the FPA model (Eq.16). Much more detailed experimental data is certainly needed, however, before these ideas can be put to the test. 


\section{The Rôle of Local Inhomogeneities}

\subsection{A STOCHASTIC WAVE EQUATION}

Provided that local conservation laws (those arising from mechanical equilibrium) are obeyed, many local rules for force transmission are a priori compatible with the existence of contacts among rigid particles [26, 27]. Therefore, even if there is a definite mean relationship among stresses at the meso-scale (as models like FPA assume), one can expect randomness in the local transmission coefficients. The simplest model for this and other sources of randomness is to introduce a randomly varying 'speed of light' $c_{0}$. This could describe the fact that, for example, the parameter $p$ in the model of figure 2 can vary from grain to grain.

This suggests the following stochastic wave equation for stress propagation in two dimensions:

$$
\partial_{t t} \sigma_{t t}=\partial_{x x}\left[c_{0}^{2}(1+v(x, t)) \sigma_{t t}\right]
$$

where $v(x, t)$ is a random noise. We have studied this equation in great details [28] using perturbation theory, and find that for weak disorder, the average response function now has two peaks of finite (diffusive) width (rather than two $\delta$ peaks in the zero disorder case), and that the 'speed of light' is renormalized to a lower value $c_{R}$. More interestingly, the unaveraged response function takes negative (and rather large) values. This may be of crucial importance since it suggests a fundamental instability of granular matter to external perturbations. Suppose indeed that as a result of a distant perturbation, a certain grain receives a negative (upward) force larger than the pre-existing downward vertical pressure. This grain will then move and a local rearrangement of contacts will occur. If stability is to be recovered, this rearrangement must induce a variation of $c_{0}(x, t)$ so as to reduce the cause of the instability. Thus, the stochastic wave equation implicitly demands rules similar to those introduced in [19] to describe extreme sensitivity to external perturbations in silos. The present model, which is purely static, does not say what happens when a local rearrangement occurs, but certainly suggests that small perturbations will induce such rearrangements. One can show that typically a perturbation of order the weight of one grain is enough to oblige rearrangements somewhere else in the pile [28]. This would cease to be true if large enough overload was applied to ensure that that all grains are subject to a vertical compression greatly in excess of their weight. But it is not clear that such an overload is ever really possible: even at the bottom of a deep pile, a finite fraction of grains may be effectively non-loadbearing [10].

We have also studied the weight-weight correlation function, in the geometry of a flat layer with a random overload at the top, neglecting gravity. 
We find [28] that, as a function of (horizontal) distance, the correlation has two peaks. The first one is of course at separation $\Delta x=0$, while the second is at $\Delta x=2 c_{R} H$, which simply means that two points at the bottom of the packing connected by 'light rays' to the same point on the top, share information about the overload. This result is of importance since the shape of this correlation function clearly differs from the corresponding one in the scalar model, which is a single peak at $\Delta x=0$. It also differs from that pertaining to a simple elastic medium, where the weight-weight correlation, in this geometry, decays only very slowly: correlations extend to the scale of the system size itself. Measuring carefully the averaged correlation function of a granular system under an overload could then confirm (or disprove) the presence of a ray-like propagation. A stress correlation function was recently measured in [10] and found to be featureless, but measurements extended only to very short lengthscales: $\Delta x \leq 5 a$, as compared to the height of the pile $H \simeq 100 a$. We thus expect the features of the correlation function to show up on much larger scales $\left(\sim 2 c_{R} H\right)$ than those measured so far.

\subsection{STRESS HISTOGRAM}

We have seen above that within a scalar approach, an exponential-like distribution (possibly of the type $\exp -w^{\beta}$, with $\beta \geq 1$ ) is expected [9, 11]. One can ask whether this exponential distribution survives within a tensorial description. So far, we only have partial numerical results, based on a direct simulation of the three-leg model introduced above, with a random $p$ chosen between 0 and $p_{M}$. This scheme is thus very close in spirit to the $q$-model. However, as emphasized above, the local vertical forces are not everywhere positive; one should thus introduce an extra rule to cope with this instability. Several possibilities come to mind, but we have not yet explored them (see however $[26,27]$ ). Nevertheless, the large force region, which is presumably not sensitive to the presence of negative forces, behaves much in the same way as in the scalar $q$-model. In particular, the tail of the distribution decays as $\exp -w^{\beta}$, with $\beta \simeq 1$ when $p_{M}=1$, and with $\beta>1$ when $p_{M}<1$. More work is needed to understand the physical implications of the presence of negative forces and any relation this may have to the static avalanche phenomenon [19]. However, the above results show that the tail of the force distribution is only exponential in a 'strong disorder' limit, where local 'arching' (i.e. one grain entirely bearing on a single downward neighbour) has a nonzero probability. 


\section{Modelling Strategies for Static Granular Media}

We now compare our approach (as outlined above) to the problem of sandpile modelling with previous ones, and address various criticisms of it that have recently been made. Specifically we are interested in calculating the stress distribution at the base of a pile constructed on a rough, rigid support. For clarity of the discussion, we again limit the mathematics to two dimensions, although we emphasize again that our work, particularly that on the FPA model, was developed in the context of three dimensional conical piles.

\subsection{LOCAL RULES FOR FORCE TRANSFER}

Above we have described an approach to the modelling of stress propagation in granular media based on local rules for the transfer of forces between grains. Broadly speaking, to leading order in a gradient expansion [21, 28] the closure (14) exhausts the possibilities for local models in which the forces passed from a grain to its neighbours in the layer below involve a linear decomposition of the "incident force" $\left(F_{x}, F_{t}\right)$, which is taken as the vector sum of forces acting on the grain from those in the layer above. Somewhat similar considerations underlie the so-called "clastic" theories of "discontinua" introduced by Trollope [29]. Indeed, some authors have confused the two models, and our approach has been criticized for "reinventing theories... that are well-known in another disciplinary area" [15].

However, Trollope's model, though linear, does not lead to Eq.14. Its distinguishing feature is instead that the vector sum of the incident forces on a grain is not taken before applying a rule to determine the outgoing forces from that grain. The outgoing forces instead depend separately on each of the incident force contributions. This feature is, in our view, strongly unphysical: if a grain is subjected to two small extra forces, whose vector sum is vertical, from its neighbours in the layer above, the force increments exerted on the grains below should be equivalent to a small increase in its weight. Within Trollope's rules, this is not the case [30, 21]. It is therefore disingenuous to criticize our approach on the grounds that 'Trol-

lope's clastic or discontinua model, that was rejected by Wittmer et al. on the grounds of being "unphysical", actually contains the FPA solution' [15]. For, although Trollope's model can be tuned to give the same stress pattern as the FPA model in a symmetric two dimensional pile, these two models have distinctly different physical content and give differing predictions in other geometries. 


\subsection{HYPERBOLIC CONTINUUM MODELS}

Although motivated in part by simple packing models (e.g. figure 2), our approach does not require such a specific microscopic interpretation and can instead by formulated in purely continuum mechanical terms [31]. This is done by postulating a constitutive relations among stresses such as (14). (The latter includes, for special values of $\mu$, both (11) and (16) which describe respectively the case of symmetric stress propagation and the FPA model.) We have noted already the existence of an alternative constitutive equation among stresses, corresponding to the Mohr-Coulomb rigid-plastic model (or IFE model) which is a widely studied continuum theory of granular media. In fact this reads (in two dimensions):

$$
\sigma_{x x}=\sigma_{z z} \frac{1}{\cos ^{2} \phi}\left[\sin ^{2} \phi+1 \pm 2 \sin \phi \sqrt{1-\left(\cot \phi \sigma_{z x} / \sigma_{z z}\right)^{2}}\right]
$$

All these approaches lead to hyperbolic equations for stress propagation.

A reasonable question [15] is to ask why we are not satisfied with the IFE closure (18). The main cause is that we can see no physical reason for it to be true. Indeed, even supporters of this rigid-plastic model do not usually propose it as an accurate description of sandpile behaviour; it is more often viewed as a way of generating certain "limit-state" solutions. In the simplest geometries these solutions correspond to taking the - or + sign in (18); these are often referred to as "active" and "passive" limitstates, respectively [32]. It is easily established [13, 21] that for a sandpile at its repose angle, only one solution of the resulting equations exists in which the sign choice is everywhere the same. This is the active solution, and it shows a hump, not a dip, in the vertical normal stress beneath the apex. Savage, however, draws attention to a "passive" solution, having a pronounced dip beneath the apex [15]. This solution actually contains a pair of matching planes between an inner region where the positive root of (18) is taken, and an outer region where the negative is chosen.

In principle this does not exhaust the repertoire of IFE solutions for the sandpile: there should exist others, with larger numbers of matching planes between segments of alternating sign [21]. In any case, the predictive power of the rigid-plastic approach largely depends on a belief that the limit-state solutions can be "generally regarded as bounds between which other states can exist, i.e., when the material is behaving in an elastic or elastoplastic manner" [15]. Unfortunately, this belief is unfounded: counterexamples exist (figure 5), even among elastoplastic models of the simplest kind [21]. Therefore the so-called limit-states should be regarded merely as "rule of thumb" estimates; these may be useful for engineering purposes, but do not shed much light on the physics of stress propagation in granular matter. 


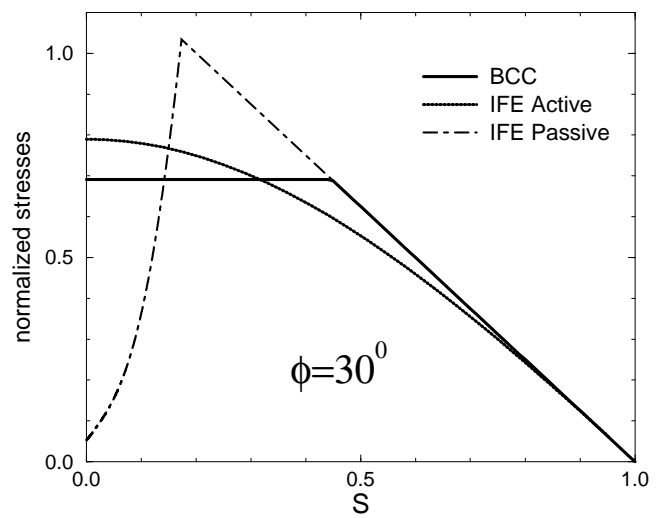

Figure 5. Vertical normal stress found from Eq.11 (the BCC model, [12]), for a pile at angle of repose $\phi=30$ degrees, compared to the active and passive IFE solutions. (The IFE solutions are obtained by shooting from the midplane for $P=\left(\sigma_{t t}+\sigma_{x x}\right) / 2$ and the polar angle $\theta$ as functions of the direction of the principal axis $\Psi$.) Note that active and passive IFE solutions do not bound the stress, either in the model of Eq.11 or in the elastoplastic model of [16], which, for a certain parameter choice, yields identical results to Eq.11 (see [16]).

\subsection{ELASTOPLASTIC MODELS}

All the models considered above make no mention of strain variables. A partial justification for this was given in Refs.[3, 13], namely that the experimental data obey radial stress-field (RSF) scaling, which implies that there is no characteristic length-scale. Since elastic deformation under gravity introduces such a length-scale (a "sagging length") the observation of RSF scaling to experimental accuracy in most but not all the data $[1,2,15]$ suggests that elastic deformation is not significant.

This does not imply that an elastic or elastoplastic description of sandpiles is impossible; but it shows that in any such description, the limit of a large elastic modulus appears to be the relevant one. This limit yields equations, in the bulk of the medium, for which strain variables cancel out; and this fact is usually exploited in elastoplastic calculations (see, e.g., [16]). Note that it is tempting, but entirely wrong, to assume that strain variables on the boundary of the medium also cancel in this limit (see below).

In fact, as correctly noted by Savage [15] results similar to those of the FPA model and the related models described above can, in two dimension (only), be obtained within such an elastoplastic modelling approach. Typically, an inner, linear elastic region is matched, by hand, onto an outer plastic one. An example of this procedure was described recently by Can- 
telaube and Goddard [16] whose approach is similar to earlier work by Samsioe [33]. This analysis can be made to give mathematically identical results to those found with some, if not all, values of $\mu$ in Eq.14.

As they stand, however, such elastoplastic analyses are devoid of physical meaning, for the following reason. Recall that the aim of the exercise is to calculate the forces measured at the base of a pile of sand. Recall also the well-known theorem that to find the equilibrium state of an elastic body, one must specify either the surface force field or the displacement field at all points on its boundary [34]. Accordingly, it is meaningless to "calculate" the forces at the base of an elastoplastic pile without specifying a boundary condition at the bottom surface of any elastic zones present. To specify as boundary conditions the forces themselves there is mathematically legitimate, but cannot really be described as a "solution" to the sandpile problem. On the other hand, to specify the displacements is (by this stage) formally impossible, since the relevant variables have already been eliminated by taking the limit of a high elastic modulus.

The only way to circumvent this difficulty is to specify the displacement field at the base first, and then take the limit of a high modulus afterwards. To obtain finite forces, the displacements must be allowed to tend to zero but, crucially, the results depend on how this limit is taken. This is illustrated in figure 6 . The challenge to elastoplastic modellers is then to decide what (infinitesimal) displacement field should be chosen at the base. For a genuine elastoplastic body, which is placed on a rough rigid support, this displacement field depends on the precise manner in which the body was brought into its state of rest. For a sandpile constructed by (say) pouring sand grainwise from a point source the problem seems much less well-posed since (despite assertions to the contrary [15]) there is no obvious physical definition of the displacement field in such a pile [35].

\subsection{ELASTIC INDETERMINACY}

The circumstances just described apply to any model of a sandpile in which the elliptic equations of elasticity are invoked in all or part of the medium. We have called this the problem of "elastic indeterminacy" [21]. Several responses to this challenge are possible. One is to assume that the elastoplastic description is essentially sound, and seek some physical procedure whereby the displacement field at the support, or some equivalent information, is determined [36]. This is certainly worth pursuing, but we suspect that any description which does not explicitly consider the construction history of the pile is very unlikely to be successful.

A second response, which appears to be that of Evesque [17] is to conclude that the experimental results are and must be indeterminate. Put 

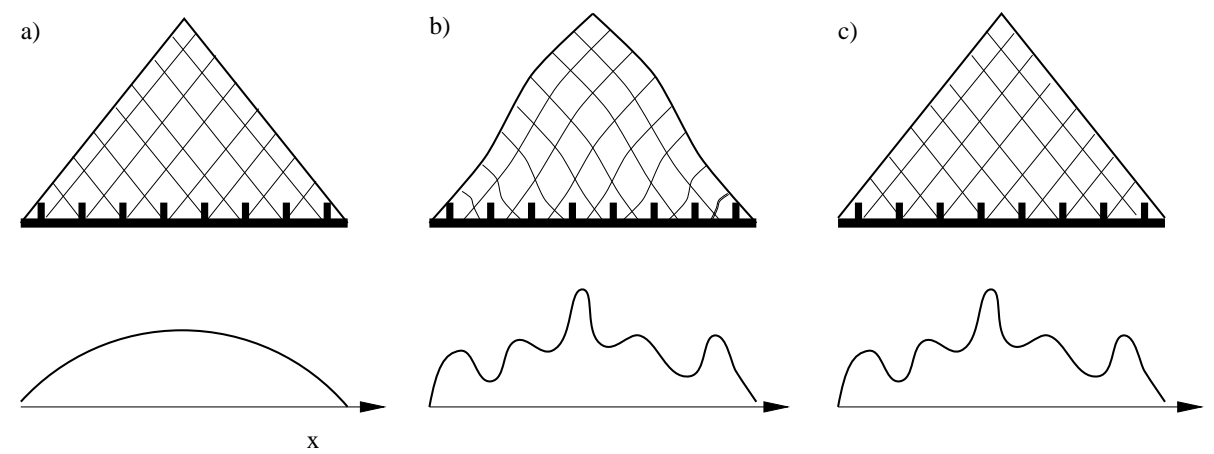

Figure 6. Consider the static equilibrium of an elastic body of finite modulus resting on a completely rough surface. Starting from any initial configuration, another can be generated by pulling and pushing parts of the body horizontally across the base (i.e., changing the displacements there); if this is rough, the new state will still be in static equilibrium. This will generate a stress distribution, across the supporting surface and within the pile, that differs from the original one. If the limit of a large modulus is now taken (at fixed stress), this procedure allows one to generate arbitrary differences in the stress distribution while generating neither finite distortions in the shape of the body, nor any forces at its free surface. An exactly analagous "elastic indeterminacy" exists in any simple elastoplastic theory of sandpiles, where an elastic zone, in contact with part of the base, is attached at matching surfaces to a plastic zone.

differently, Evesque argues that the external forces acting on the base of a pile can in fact be varied at will by the experimentalist. Certainly, if sandpiles obey Hooke's law, he must be right: for an elastic body, the experimentalist is free to prod about at the base of a pile in such a way as to change arbitrarily the forces acting there. (Moreover, in the large modulus limit, only infinitesimal proddings are required.)

In contrast to this, experimental reports clearly suggest $[1,2]$ that the forces on the base can be measured more or less reproducibly, and (though subject to statistical fluctuations) do not vary too much from one pile to another. Moreover, the experimental data for the forces show RSF scaling at the base; this is confirmed by comparing data from piles of different heights. Within an elastoplastic framework, this scaling should be violated by the (arbitrary) boundary forces at the base and hence can only be expected in the upper extremity of the pile $[16,21]$.

All this suggests a third response to the challenge of elastic indeterminacy. This is to argue that Hooke's law has very little relevance to the mechanics of sandpiles and that models based on local force propagation rules among grains are far closer to the real physics of the problem. The models we have developed along these lines give hyperbolic equations for stress propagation, and in doing so contradict Hooke's law in a fundamental 
way. This applies even in their incremental response to small added loads. Whether this is a drawback or an advantage depends on one's view of the physics. Certainly, if one believes that any granular assembly must behave elastically under sufficiently small incremental loads, then models such as ours can only describe the behaviour beyond some finite threshold [36]. (We are as yet unconvinced of whether this threshold is finite; for example it might vanish in the high modulus limit.) In any case, in calculating the response to gravity itself we believe that any such threshold is easily exceeded throughout the pile, and hence that our approach has far more to offer than models invoking elastic deformations from some hypothetical unstrained (i.e., zero gravity) state. On the other hand, the existence of a threshold might make it somewhat harder to detect experimentally the response and correlation functions $[12,13,28]$ which are, as described above, strong signatures of hyperbolic stress propagation laws.

The varying perspectives laid out above are not necessarily completely contradictory, in that the global ('coarse-grained') features of the stress pattern could be governed by determinate equations but the details not. As well as being subject to elastic indeterminacy, the latter could be affected by randomness in the grain packings, which may be exquisitely sensitive to temperature and other poorly-controlled parameters [19].

\section{Boundary Conditions in Hyperbolic Models}

We now consider more carefully the role of boundary conditions in our approach. In contrast to the elliptic equations of elasticity, the hyperbolic equations arising from constitutive relations among stresses admit definite solutions for the stresses at the base of a freestanding pile. By the same token, if one tries to apply all the boundary conditions appropriate to an elastic body (e.g. specifying the surface forces acting over the entire surface), then in general no solution will exist of the hyperbolic equations that pertain to any particular choice of constitutive relation. We discuss these two aspects in turn.

\subsection{DETERMINACY OF THE SANDPILE}

The procedure for a sandpile is of course to specify zero-force boundary conditions at the free (upper) surfaces. Within our description, the response arising from a localized body force (a "source term" in the equations) propagates downward along two characteristics passing through the source. In models obeying (14) these characteristics are, in addition, straight lines. The force on the base is found simply by summing the contributions from all the body forces; this is a fully determinate procedure for any closed set of hyperbolic equations $[12,3,13]$. Note that in principle, one could 
envisage propagation also along the "backward" characteristics (see figure $7(\mathrm{a}))$. This is forbidden since these cut the free surface; any such propagation can only arise in the presence of a nonzero surface force, in violation of the boundary conditions. Therefore the fact that propagation occurs only along downward characteristics is not related to the fact that gravity acts downward; it arises because we know what forces act at the free surface (the forces there are zero). Suppose we had instead an inverse problem: a pile or bed with some unspecified overload at the upper surface, for which the forces acting at the base had been measured. In this case, the information from the known forces could be propagated along the upward characteristics to find the unknown overload.

(a)

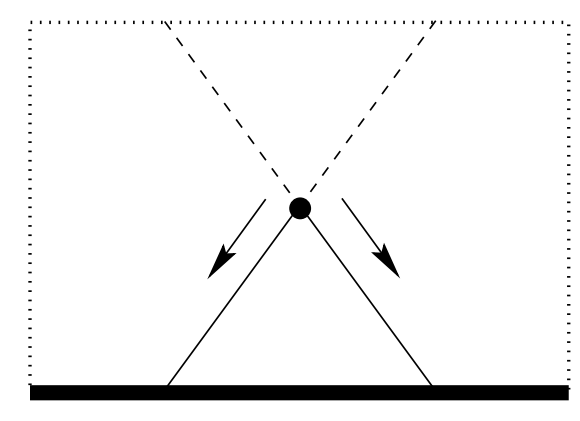

(b)

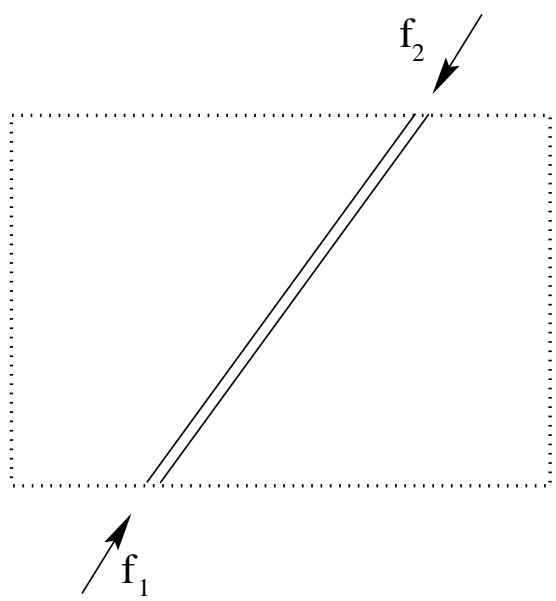

Figure \%. (a) The response to a localized force is found by resolving it along characteristics through the point of application, propagating along those which do not cut a surface on which the relevant force component is specified. For a pile under gravity, propagation is only along the downward rays. (b) Admissible boundary conditions cannot specify separately the force component at both ends of the same characteristic. If these forces are unbalanced (after allowing for body forces), static equilibrium is lost.

\subsection{STRESS PATHS}

Informally speaking, the hyperbolic problem is determined once half of the boundary forces are specified. More precisely (figure 7(b)) one is required to specify the surface force tangential to each characteristic ray, at one end and one end only. The corresponding force acting at the other end is obliged to balance it, allowing for any body forces acting tangentially along the ray. If it does not do so, then within our modelling approach, the material ceases to be in static equilibrium. This is no different from the 
corresponding statement for a fluid or liquid crystal; if boundary conditions are applied that violate the conditions for static equilibrium, some sort of motion results. Unlike a fluid, however, for a granular medium we expect such motion to be in the form of a finite rearrangement rather than a steady flow. Such a rearrangement will change the microtexture of the material, and thereby alter the constitutive relation among stresses. We expect it to do so in such a way that the new constitutive relation is compatible with the imposed forces.

Although simplified, we believe that this picture correctly captures some of the essential physics of stress paths. Such paths are load-bearing structures within the contact network and, in the simplest approximation of straight, unbranched paths, these must have the property described above (the forces on two ends of a path must balance). Stress paths should therefore be identified (on the average) with the characteristics of our hyperbolic equations. The mean orientation of the stress paths is then reflected in a constitutive equation such as (11) or (16).

The physics of our modelling approach is thus to assume that the mean orientation of stress paths, in each element the material, is fixed at burial. (This does not necessarily require that the individual paths are themselves fixed.) This was called earlier the "perfect memory assumption". We think it reasonable to assume that the stress paths will not change their average orientations so long as they are able to support the applied load. But if a load is applied which they cannot support, rearrangement is inevitable [17]. This causes some part of the pile, at least, to adopt a new microtexture and thereby a new constitutive relation. In other words, loadings of this kind cause the construction history of the pile to change.

\section{Conclusion: Sandpiles as Fragile Matter}

Within our modelling approach, a granular assembly is able to support some, but not all, of the surface loads that would be supportable by an elastic continuum. Such models may therefore provide an interesting paradigm for the behaviour of "fragile matter", a concept which may be useful in other systems where certain combinations of applied forces, even if small, are enough to force irreversible reconstruction of the material. (Such systems could include a number of disordered soft solid materials such as defect textures in liquid crystals.)

In the present context, fragility arises from the the requirement of tangential force balance along stress paths. If this is violated at the boundary, even infinitesimally, then internal rearrangement must occur, causing new stress paths to form, so as to support the load [37]. Obviously, this might be rather too simple a picture - for example, branching of stress paths is 
ignored. Thus it remains possible that Hookean behaviour is recovered for sufficiently small perturbing forces [36, 17]. However, for practical purposes we believe our approach, by capturing at least some of the physics of stress paths, may have rather more to offer than ideas based on the physics of conventional (homogenous, isotropic) elastic, elastoplastic or rigid-plastic media. Phenomena that seem to be addressable in such terms include that of arching and, in its various aspects, the role of noise.

Clearly, there is much scope for developing these models further. In particular it would be very useful to have an understanding of the crossover, if one indeed exists, between fragile and elastic regimes: the latter should ultimately be restored in a sufficiently large pile (beyond the "sagging" length). Equally important is to confront these and other modelling approaches with much more demanding experimental tests, of which several were suggested above.

\section{Acknowledgements}

We are grateful to P. Evesque, J. Goddard, J. Jenkins, S. Savage and F. Radjai for discussions, and to H. Herrmann for facilitating some of these during the present School. MEC acknowledges the hospitality of ITP Santa Barbara where some of these ideas were developed during discussions with S. Edwards, D. Levine, S. Nagel, C. Thornton, T. Witten and other participants of the "Jamming and Rheology" programme. This research was funded in part by EPSRC (UK) Grants GR/K56223 and GR/K76733. 


\section{References}

1. J. Smid and J. Novosad, Proc. of 1981 Powtech Conference, Ind. Chem. Eng. Symp. 63, D3V 1-12 (1981).

2. R. Brockbank, J.M. Huntley, and R.C. Ball, J. Phys. II (France), 7, 1521-1532 (1997).

3. J.P. Wittmer, M.E. Cates, P. Claudin and J.-P. Bouchaud, Nature (London) 382, 336 (1996).

4. R.L. Brown and J.C. Richard, 'Principles of Powder Mechanics' (Pergamon, New York, 1966).

5. L. Vanel, E. Clément, J. Lanuza and J. Duran, preprint submitted to Phys. Rev. Lett.; and this volume.

6. F. Radjai, M. Jean, J.-J. Moreau and S. Roux, Phys. Rev. Lett. 77274 (1996); F. Radjai, D.E. Wolf, M. Jean and J.-J. Moreau, Binomal character of stress transmission in granular packings, preprint; and this volume.

7. G.W. Baxter, in Powders and Grains 97, Behringer and Jenkins eds., Balkema, Rotterdam (1997).

8. see e.g. P. Dantu, Proc. of the fourth Int. Conf. On Soil Mech. and Found. Eng. (London 1957), 1 144, Annales des Ponts et Chaussées, IV, 193 (1967), T. Travers et al., J. Phys. France, 49939 (1988).

9. C.-H. Liu, S.R. Nagel, D.A. Scheeter, S.N. Coppersmith, S. Majumdar, O. Narayan and T.A. Witten, Science 269, 513 (1995).

10. D.M. Mueth, H.M. Jaeger and S.R. Nagel, preprint

11. S.N. Coppersmith, C.-h. Liu, S. Majumdar, O. Narayan and T.A. Witten, Phys. Rev. E 53, 4673 (1996).

12. J.-P. Bouchaud, M.E. Cates and P. Claudin, J. Phys. I (France) 5, 639 (1995).

13. J.P. Wittmer, M.E. Cates and P. Claudin, J. Phys. I (France) 7, 39 (1997).

14. See, e.g., G. Gudehus in Powders and Grains 97, Behringer and Jenkins eds., Balkema, Rotterdam (1997), p. 169-183.

15. S.B. Savage in Powders and Grains 97, Behringer and Jenkins eds., Balkema, Rotterdam (1997), p. 185-194; see also New Scientist, 2083, p.28 (1997).

16. F. Cantelaube and J.D. Goddard, in Powders and Grains 97, Behringer and Jenkins eds., Balkema, Rotterdam (1997), p231-234.

17. P. Evesque and S. Boufellouh, in Powders and Grains 97, Behringer and Jenkins eds., Balkema, Rotterdam (1997) p.295-298; P. Evesque, J. Physique I, 7, 1305-1308 (1997); P. Evesque, private communication.

18. S.F. Edwards and R.B. Oakeshott, Physica D 38, 88-93 (1989); see also S.F. Edwards and C.C. Mounfield, Physica A 226, 1,12,25 (1996).

19. P. Claudin and J.-P. Bouchaud, Phys. Rev. Lett. 78, 231 (1997); and this volume.

20. H.A. Janssen, Z. Vert. Dt. Ing. 39, 1045 (1895); see also R.M. Nedderman, Statics and Kinematics of Granular Materials, Cambridge University Press (1992).

21. M.E. Cates, J.P. Wittmer, J.-P. Bouchaud and P. Claudin, manuscripts in preparation.

22. For a more general geometry (without axial symmetry) at least two further closure relations are required. Possibilities include not only isotropic, wavelike propagation (in $2+1$ dimensions), but also propagation along three characteristic rays in the form of a tripod. The latter is perhaps the most natural extension of the FPA hypothesis to arbitrary geometries.

23. F.H. Hummel and E.J. Finnan, Proc. Inst, Civil Eng. 212, 369-392 (1920).

24. I.F. Lee and J.R. Herington, Proc. 1st Aust.-N.Z. Conf. Geomech., 1, 291-297 (1971).

25. P. Evesque, Phys. Rev. A 43, 2720 (1991); P. Evesque, D. Fargeix, P. Habib, M. P. Luong and P. Porion, Phys, Rev. E 47, 2326-2332 (1993).

26. C. Eloy and E. Clément, to appear in J. Phys. I (France) (1997).

27. J. Socolar, preprint, cond-mat/9710089.

28. P. Claudin, J.-P. Bouchaud, M.E. Cates and J. Wittmer, preprint, cond- 
mat/9710100, submitted to Phys. Rev. E.

29. D.H. Trollope, in Rock Mechanics in Engineering Practice, pp. 275-320, K.G. Stagg and O.C. Zienkiewicz, (Eds.), (Wiley, New York, 1968). D.H. Trollope and B.C.Burman, Géotechnique 30, 137-157 (1980).

30. For nonzero values of his arching parameter $k$. For $k=0$, the continuum limit of Trollope's model coincides with 11. However, this is not the case discussed by Savage [15].

31. It would be a mistake to suggest that our modelling strategy is fundamentally at odds with continuum-mechanical principles [16] (or even with the laws of newtonian mechanics themselves [17]). Such remarks seem to be based on the idea that Hooke's law is implicit in any continuum (or even newtonian) description. This is untrue, as the existence of successful continuum theories of fluids and liquid crystals shows. We return below (Section 7.4) to the issue of whether Hooke's law can usefully be applied to granular media under gravity.

32. Our definitions of "active" and "passive" are not quite the same as those used elsewhere in the literature, in which the terms refer to global properties of the solutions rather than the choice of a local constitutive equation. According to the latter, Savage's identification of the $(+/-)$ solution containing a matching plane as everywhere passive is correct, although in our terminology it a solution with an active outer and passive inner region.

33. A.F. Samsioe, Géotechnique 5, 200-223 (1955).

34. See, e.g., L.D. Landau and E.M. Lifshitz, Theory of Elasticity, 3rd Edn., Pergamon, Oxford 1986, or G. E. Mase, Continuum Mechanics, McGraw Hill, NY 1970.

35. Since a sandpile would not exist at all in the absence of gravity, it is not clear whether one can as usual define displacements relative to a reference state in which no body or surface forces act.

36. We are grateful to Tom Witten and others for discussions on this point.

37. There is a sense in which this can be viewed as "incipient failure everywhere", except that the failure in question is not Mohr-Coulomb, but instead connected with the failure of stress paths under imbalanced tangential loads. 